\title{
Bienestar Laboral: Diferencias entre Empresas Sociales, Entidades Públicas y Empresas Capitalistas en España
}

\author{
Esther Villajos ${ }^{1}$, Núria Tordera² \& José M. Peiró ${ }^{3}$
}

Recibido: 28/05/2019 Aceptado: 23/10/20129

DOI: $10.21772 /$ ripo.v38n2a04

\begin{abstract}
Resumen
El bienestar de los trabajadores se ha convertido en uno de los resultados organizacionales más importantes. La investigación en este campo se focaliza tradicionalmente en empresas capitalistas, pero las organizaciones pueden presentar distintas formas jurídicas. Nuestro objetivo es analizar si la forma jurídica juega un papel diferencial en el bienestar de los trabajadores, operacionalizado con distintos indicadores. Los resultados revelaron diferencias en cuanto a los niveles de bienestar en tres tipos de organizaciones: empresas sociales, entidades públicas y empresas capitalistas. En trabajadores general, los de las empresas sociales presentan un mayor grado de bienestar en la mayor parte de indicadores. Sin embargo, cuando el bienestar se refiere a la satisfacción extrínseca o la orientación al bienestar hedónico, estos son mayores para los trabajadores de las empresas capitalistas.
\end{abstract}

Palabras clave: Bienestar laboral, Orientación a la felicidad, Forma jurídica, Empresas sociales, Empresas capitalistas, Empresas públicas.

\section{Well-being at Work: Differences among Social Enterprises, Public Entities and Capitalist Enterprises}

\begin{abstract}
Employees' well-being has become one of the most important organizational outcomes. This research has traditionally focused on companies with a lucrative nature, but organizations can present different legal types. Our goal is to analyse whether the legal type plays a differential role on employees' well-being, operationalised with different indicators. The results revealed differences in the levels of well-being among the three types of organizations: social, public and for-profit organizations. In general, employees from social enterprises showed a greater degree of well-being in most indicators. However, when well-being is measured as extrinsic satisfaction or hedonic well-being orientation, these are higher for employees in for-profit companies.
\end{abstract}

Key words: Well-being at work, Happiness orientation, Legal type, Social enterprises, Public entities, Capitalist enterprises.

\footnotetext{
1 Dra. En Psicología. Universidad Internacional de Valencia. Profesora docente investigadora. ORCID: https://orcid.org/0000-0003-2815-8478 E-mail: Esther.villajos@uv.es

2 Dra. en psicología y profesora titular en el departamento de psicología social de la Universitat de València. ORCID: https://orcid.org/0000-0003-28158478 E-mail: Nuria.tordera@uv.es

3 Dr. Profesor catedrático. Universitat de València; IVIE (Instituto Valenciano de Investigaciones Económicas).ORCID: https://orcid.org/0000-00015293-0355 E-mail: Jose.M.Peiro@uv.es

Cómo citar este artículo: Villajos, E. Tordera, N. \& Peiró, J. M. (2019). Bienestar Laboral: Diferencias entre Empresas Sociales, Entidades Públicas y Empresas Capitalistas en España. Revista Interamericana de Psicología Ocupacional, 38(2), 85-103. DOI: 10.21772/ripo.v38n2a04
} 


\section{Introducción}

La mayor parte de la investigación en el ámbito de la gestión de recursos humanos, se ha hecho desde una lógica basada en la realidad empresarial de las organizaciones capitalistas o con ánimo de lucro. Por este motivo, un objetivo central ha sido la determinación de prácticas y políticas de recursos humanos que permitieran incrementar el desempeño o la productividad de los trabajadores. Algunos autores han incidido en la necesidad de acercarse a la gestión desde otras lógicas. En este sentido se ha señalado la necesidad de centrarse no solo en objetivos económicos sino también sociales y humanos (Paauwe, 2004; Guest, 2017). Siguiendo esta perspectiva, el bienestar de los trabajadores se ha incorporado a la investigación sobre gestión de los recursos humanos, convirtiéndose en uno de los resultados organizacionales que más atención ha recibido en los últimos años (Van de Voorde, Paauwe, y Van Veldhoven, 2012; Peiró, Ayala, Tordera, Lorente y Rodríguez. 2014). Aunque este nicho de investigación está aumentando, la mayor parte de la investigación se sigue realizando con muestras de empresas con ánimo de lucro, convirtiéndose en una práctica habitual en la investigación en gestión de recursos humanos.

La comparación entre formas jurídicas o tipos de organizaciones se ha focalizado, tradicionalmente, en diferencias de gestión y productividad entre entidades públicas y empresas con ánimo de lucro. Estudiar estas diferencias ha estado en la agenda de investigación durante años sobre todo marcado por el interés, durante los años 90, de aplicar prácticas y políticas de empresas con ánimo de lucro al sector público (Kalleberg, Marsden, Reynolds, y Knoke, 2006). Aun así, dadas las diferencias existentes entre estas dos formas jurídicas resulta difícil aplicar gestiones similares (Vandenabeele, Brewer, y Ritz, 2014) puesto que los objetivos entre ambas difieren considerablemente. Además, esta comparativa no ha incluido, generalmente, otro tipo de organizaciones como las empresas sociales. Éstas son organizaciones que tienen un claro impacto económico y social y que, por sus características específicas difieren de las anteriores (Doherty, et al., 2014). Son una parte importante del tejido productivo y de servicios y juegan un papel muy importante en el desarrollo del estado del bienestar de las naciones.

Cada vez es mayor la investigación que plantea la necesidad de afrontar una aproximación al estudio de la gestión de organizaciones y sus recursos humanos que considere el contexto en el que se desarrolla dicha gestión (p.ej., país, sector, tipo de organización ...) (Paauwe, 2014). En este sentido, la teoría institucional plantea que las diferentes influencias internas y externas a las que están sujetas distintas organizaciones por cuestiones como, por ejemplo, la forma jurídica puede generar las consecuencias que este hecho puede tener sobre diversos resultados, tales como el desempeño o el bienestar de los mismos. En este trabajo se parte de esta aproximación teórica para entender como las empresas con ánimo de lucro o capitalistas, las entidades públicas y las empresas sociales responden a lógicas diferentes, tienen diferentes objetivos, valores y estructuras, y como estas diferencias pueden afectar al nivel de bienestar que presentan sus trabajadores.

El objetivo de este trabajo es, por lo tanto, analizar las diferencias en términos culturales y de gestión de organizaciones con distintas forma jurídica de las organizaciones (ánimo de lucro o capitalistas, pública y social) y su relación con los niveles de bienestar experimentados por los trabajadores (según distintos indicadores). Asimismo, se analizan las diferencias en la orientación hacia el bienestar de los trabajadores en los tres tipos de organizaciones.

En el próximo apartado se define el concepto de bienestar, la importancia de su estudio en entornos laborales y los indicadores relevantes para su estudio. Seguidamente, se analizan las diferencias culturales y de gestión entre los tres tipos de formas jurídicas. Finalmente, se 
presentan los análisis, resultados, la discusión y las conclusiones del presente estudio.

\section{Bienestar laboral}

La investigación sobre el bienestar laboral es un tema central en el ámbito de la psicología organizacional y del trabajo. El bienestar de los trabajadores se considera una variable clave en el estudio de aspectos laborales como por ejemplo el desempeño laboral (Judge, Thoresen, Bono, y Patton, 2001), la rotación de los trabajadores (Irvine y Evans, 1995) o el burnout o síndrome de desgaste profesional (Tarcan, Tarcan, y Top, 2017) entre otros.

El análisis del bienestar se ha llevado a cabo desde distintas perspectivas (Warr, 2013). A pesar de que la mayor parte de estudios se centran en el concepto de satisfacción laboral, varios autores han puesto de manifiesto la necesidad de ampliar su estudio a otras conceptualizaciones tales como el afecto positivo, la satisfacción con la vida o la salud física.

De entre las distintas conceptualizaciones, una diferenciación clave es la establecida entre el bienestar hedónico y el eudaimónico. El bienestar hedónico se caracteriza por centrarse en el bienestar como placer, como sensación tanto física como mental centrada en el bienestar individual de manera agradable o placiente y en ausencia de dolor(Ryan y Deci, 2001). En la literatura científica sobre el ámbito laboral, este tipo de bienestar es el más recurrente, se le conoce como bienestar subjetivo (Diener y Lucas, 1999) y generalmente se ha operacionalizado como satisfacción laboral (Petrescu y Simmons, 2008). La comprensión del bienestar hedónico de los trabajadores requiere ampliar el espectro del bienestar laboral a otros ámbitos no relacionados con el trabajo, como por ejemplo la satisfacción con la vida (Guest, 2002; Warr, 2013). Para entender el impacto del entorno laboral en el bienestar, es imprescindible atender a este indicador puesto que una buena parte de la vida acontece en el marco laboral, y muchas de las satisfacciones e insatisfacciones del trabajo repercuten en la vida. No obstante, otros investigadores han señalado la importancia de considerar también medidas genéricas (contextfree) frente a aquellas más específicas (domainspecific o job-related) (Warr y Nielsen, 2018). En este sentido, la satisfacción con la vida resulta una variable destacada a estudiar (Della Torre, 2012) dentro del bienestar hedónico, aunque ha sido olvidada en la literatura sobre gestión de recursos humanos durante mucho de tiempo.

El bienestar eudaimónico, por su parte, tiene una conceptualización más próxima a la consecución del verdadero potencial de las personas (Ryff, 1989) y resulta una variable clave, complementaria al bienestar hedónico, para tener una imagen clara del bienestar general de los trabajadores. Esta, no está tan centrada en el placer físico, sino en aspectos más psicológicos, más arraigados al crecimiento personal (Ryan y Deci, 2001) y al incremento del potencial humano. En los últimos años, desde el ámbito de la psicología positiva, se han realizado varias propuestas sobre como conceptualizar y medir el bienestar eudaimónico. Carol Ryff define el mismo en términos de desarrollo y autorrealización del individuo, y propone un modelo en el que destaca 6 componentes: propósito en la vida, autoaceptación, dominio del entorno, relaciones positivas, autonomía, y crecimiento personal (Ryff y Singer, 2008), de los cuales destaca el propósito en la vida y el crecimiento personal como elementos centrales.

La investigación actual ha mostrado la diferenciación entre el bienestar hedónico y eudaimónico como aspectos diferentes y complementarios del bienestar (Huta, 2015). Así pues, es importante considerar simultáneamente tanto medidas de bienestar hedónico como medidas de bienestar eudaimónico para tener una imagen más completa del alcance del bienestar. 
En el contexto del estudio del bienestar hedónico y eudaimónico y sus antecedentes, los valores humanos y concretamente las orientaciones hacia el bienestar de las personas han sido considerados como importantes caminos hacia el mismo. Las orientaciones hacen referencia a las motivaciones y prioridades que tienen las personas respecto a su forma de vivir, $y$ generalmente se ha distinguido entre una orientación más hedónica o más eudaimónica (Huta y Waterman, 2014). Estas orientaciones pueden tener un mayor o menor efecto en el bienestar correspondiente (Henderson, Knight y Richardson, 2014) y, por lo tanto, también resulta interesante tenerlas en consideración.

Finalmente, cabe destacar que normalmente el bienestar en el trabajo se ha analizado mediante autoevaluaciones (Ryff, 1989) puesto que las propias personas que lo experimentan se consideran los mejores informadores. Aun así, y para evitar problemas de varianza del método común, es también interesante incluir otras fuentes, como por ejemplo del supervisor o de los compañeros. Conocer la evaluación que hacen otras personas del bienestar de los trabajadores (como por ejemplo los supervisores en el ámbito laboral) puede ser relevante para tratar de entender las relaciones con otros constructos.

Con todo esto, vemos una amalgama de operacionalizaciones del bienestar que van más allá de la normalmente utilizada satisfacción laboral, y que resultan igualmente importantes de analizar y contextualizar.

\section{Diferencias culturales y de gestión en función de la forma jurídica: la importancia del contexto}

Generalmente, la mayor parte de los estudios que se centran en el bienestar laboral utilizan muestras de trabajadores de empresas multinacionales o empresas con ánimo de lucro, y sobre todo de países anglosajones, cosa que hace plantearnos si las relaciones positivas previamente establecidas entre factores organizacionales (como las prácticas de recursos humanos) y el bienestar serían extrapolables a otras poblaciones (Den Hartog, Boselie, y Paauwe, 2004).

Dentro del contexto y teniendo en cuenta otras poblaciones, un elemento claramente diferenciador lo podemos encontrar en el tipo de forma jurídica que presentan las organizaciones. Las empresas con ánimo de lucro, públicas y sociales, difieren en varios aspectos tales como sus principales objetivos o su cultura. Del mismo modo, sus trabajadores pueden tener diferentes expectativas y valores que los hayan orientado a trabajar en un tipo de organización u otro. Además, la propia interacción en el marco de la cultura organizacional lleva, mediante la influencia de esa cultura organizacional y la socialización, a influir en los valores y comportamientos de los miembros de la organización. Así pues, habría que esperar que los trabajadores presenten diferentes orientaciones y niveles de bienestar para las tres formas jurídicas que a continuación analizamos, pero también según las diferentes operacionalizaciones del bienestar que tengamos en consideración.

El papel de las variables contextuales, como por ejemplo el tipo de organización, se ha evidenciado en los últimos años como una variable clave para entender la relación entre distintos aspectos de gestión y los resultados organizativos (Paauwe, 2004). El contexto organizacional se ha definido como los procesos de gestión, la cultura organizativa y los sistemas que existen dentro de las organizaciones, y que juega un papel fundamental en la efectividad de los trabajadores (Doolen, Hacker y Van Aken, 2003). El tipo de forma jurídica, si bien corresponde a una nomenclatura legal, se utiliza para diferenciar tipos de organizaciones con culturas, sistemas y procesos de gestión diferentes, como por ejemplo las sociedades mercantiles con ánimo de lucro o las entidades públicas. 
En la medida en que la forma jurídica de las organizaciones puede ser una variable contextual relevante, el hábito de utilizar normalmente empresas con ánimo de lucro en la investigación sobre management o dirección de recursos humanos, rendimiento y bienestar ha sido criticado por anteriores autores (Aycan, Kanungo, Mendonca, Yu, Deller, Stahl y Kurshid, 2000), poniendo de manifiesto la necesidad de incluir otro tipo de organizaciones (Feeney y Rainey, 2010).

La teoría institucional (DiMaggio y Powell, 1983) nos ayuda a explicar cómo podemos clasificar a las organizaciones por tipos parecidos, según características y valores similares. Cada tipo de organización posee unas normas y regulaciones, unos actores clave y unas expectativas sociales que las diferencian.

Esta identidad institucional ayuda a entender el marco en el que se desarrollan las relaciones entre las organizaciones y los trabajadores. También tiene en cuenta las demandas internas y externas que las constriñen y posibilitan, como por ejemplo las expectativas de la sociedad y de los propios trabajadores sobre las normas y comportamientos que influirán en cada tipo de organización. Así pues, desde esta aproximación se enfatiza la importancia del ajuste vertical o estratégico y del horizontal o de ocupación (Boon, den Hartog, Boselie, y Paauwe, 2011) donde las políticas y las prácticas de gestión tienen que estar en concordancia con la estrategia y la racionalidad de las organizaciones.

Una de las principales fuentes de concordancia entre políticas y prácticas de gestión y las organizaciones son los objetivos de éstas. Es decir, según los objetivos primordiales de las organizaciones (más relacionados con la maximización del beneficio o del bienestar para la sociedad), tanto las prácticas como las expectativas de los trabajadores y de la sociedad irán encaminados a la consecución de esos objetivos.
Dentro de la institucionalización, la cultura organizativa es otro aspecto importante a tener en cuenta a la hora de analizar el tipo de forma jurídica, el contexto y el bienestar. La cultura organizativa se refiere a los valores y creencias compartidas en una organización (Day, Kelloway, y Hurrell, 2014) y es un antecedente importante de las políticas y prácticas de recursos humanos (Ostroff, Kinicki, y Tamkins, 2003) que tendrán un efecto directo o indirecto sobre el bienestar de los trabajadores. El modelo de la cultura ajustada (o culture fit en inglés) (Aycan, Kanungo, y Sinha, 1999) explica cómo, diferentes valores sociales y culturales presentes en las organizaciones y los diferentes entornos que podemos encontrar en ellas, afectarán a la cultura de trabajo interna $\mathrm{y}$, finalmente, a las prácticas implementadas. Este modelo, ya se ha utilizado en previas comparaciones contextuales, por ejemplo, para comparar organizaciones en países desarrollados y en vías de desarrollo (Kanungo y Aycan, 1998). Este modelo asume que diferentes tipos de forma jurídica (en este caso, empresas con ánimo de lucro, públicas y sociales) tendrán valores y culturas diferentes y que esto afectará a la gestión de sus recursos humanos. Además, esto también afectará a los resultados de los empleados, puesto que la cultura influye, por ejemplo, sobre la satisfacción laboral de los trabajadores (Medina, 2012).

Además, habría que esperar que estas diferencias culturales también generen diferencias en las características, expectativas y aspiraciones de los trabajadores. Así, por ejemplo, según el modelo de atracción, selección y atrición (Schneider, Smith y Paul, 2001) (ASA: Attraction, Selection, Attrition en inglés), las personas que se sienten más atraídas por la organización, que son seleccionadas con mayor probabilidad y que menos rotación presentan, serán aquellas que más comparten sus objetivos y formas de trabajo. Así, con el tiempo, las organizaciones tienden a tener trabajadores con personalidades más homogéneas (Schneider, et al., 2001) y alineados con los 
valores organizacionales. Por lo tanto, habría que esperar que las diferencias existentes entre las tres formas jurídicas en términos de objetivos, cultura, y expectativas externas e internas y estilos de gestión, también se manifiesten en sus niveles de bienestar.

Para analizar las diferencias entre los tres tipos de forma jurídica partimos del marco de análisis propuesto por Doherty, Haugh y Lyon (2014), donde se analizan la misión, la financiación y la gestión de recursos humanos que tienen estos tres tipos de organizaciones, relacionándolo con las implicaciones que pueden tener para el bienestar de sus trabajadores.

\section{Empresas con ánimo de lucro, entidades públicas y empresas sociales}

Las empresas con ánimo de lucro siguen objetivos impulsados por el mercado. Por lo tanto, su misión primordial se basa en la consecución de unos objetivos económicos o financieros. $\mathrm{Su}$ financiación se obtiene, sobre todo, de los productos o servicios generados y adquiridos por la sociedad. Esto hace que tengan que competir con sus productos o servicios y con su mano de obra, por lo que la gestión de recursos humanos seguirá también una lógica de mercado.

Es por ello que en este tipo de organización toma partido de una manera esencial la maximización de las inversiones y la distribución de dividendos o resultados. Esto hace que el bienestar extrínseco, promovido con prácticas como por ejemplo los incentivos financieros o las oportunidades de promoción sean más patentes en las empresas con ánimo de lucro que en las públicas por ejemplo (Gkorezis y Petridou, 2012). No obstante, el hecho de estar constantemente compitiendo en el mercado o con otros trabajadores (por diferentes tipos de recompensas) puede hacer que estos se sientan con menos bienestar, o con más estrés o ansiedad para tener unos objetivos marcadamente exigentes en términos de resultados. En este sentido, organizaciones o tareas que responden a una competitividad más aguda entre trabajadores, provocan niveles más altos de estrés en los mismos (Fletcher, Mayor, y Davis, 2008; Songfa, Idan, David, Ebstein, y Chew, 2018). Algunos trabajos anteriores han puesto de manifiesto que las empresas con ánimo de lucro, comparadas con las públicas presentan niveles superiores de estrés o burnout (Cottini y Lucifora, 2013). No obstante, otras investigaciones no han encontrado resultados conclusivos entre públicas y privadas según los niveles de estrés (Macklin, Smith, y Dollard, 2006), por lo que es necesario arrojar más luz sobre esta comparativa para establecer diferencias en los niveles de bienestar.

La misión de las entidades públicas es servir al beneficio colectivo. Están diseñadas para proveer de servicios públicos a la población y por tanto su objetivo primordial no es la consecución de beneficios económicos, sino sociales para el bienestar de la sociedad (Wright y Pandey, 2011). Así, su gestión económica se basa en presupuestos públicos. En función de las necesidades más imperiosas a cubrir, se estructuran los gastos y se liquidan los presupuestos. Por lo tanto, no hay beneficios económicos a distribuir entre los gerentes o trabajadores, pero la mayor o menor eficiencia influye en la eficacia de una mayor universalidad en la prestación de los servicios.

Uno de los problemas que presentan las entidades públicas sobre la gestión de los recursos humanos, es el limitado margen de maniobra que tienen sus gestores. Muchas de las prácticas vienen dictadas por la legislación, como la selección a base de oposiciones, incluso con cuotas sociales, o la dificultad de despido, por ejemplo. Otros stakeholders, como los partidos políticos, juegan también un papel muy importante en la gestión de este tipo de organización. Aun así, de acuerdo con el modelo de atracción, selección y atrición, habría que esperar que las personas que eligen trabajar en las entidades públicas coincidan con los valores que representan. Así, estas organizaciones se caracterizan por una alta 
orientación de sus trabajadores hacia la motivación al servicio público (Vandenabeele, et al., 2014), que puede estar relacionada con un bienestar más eudaimónico o de realización personal y no tanto sobre recompensas materiales, ya que suelen estar más restringidas. Además, la continuidad laboral de que disfrutan los trabajadores públicos ayuda a garantizar su confianza y compromiso con la organización a través del tiempo. De hecho, estudios recientes demuestran cómo las entidades públicas tienen trabajadores menos insatisfechos que los de las empresas con ánimo de lucro (Parent-Thirion, Fernández, Hurley, y Vermeylen, 2007). Por el contrario, esta interacción con el público o la sociedad en general, que es mucho más elevada que en las empresas con ánimo de lucro, puede llevar también consecuencias negativas relacionadas con estar más expuestos a la violencia o a las amenazas (Parent-Thirion, Fernández, Hurley y Vermeylen, 2007).

Por otra parte, la alta orientación hacia una cultura caracterizada por altos niveles de burocratización puede producir más uniformidad y un trabajo altamente controlado y organizado (Cameron y Quinn, 2011). Esto puede llevar a un menor bienestar de los trabajadores que deseen más autonomía o de los gestores que se ven en situaciones de mayores restricciones (red tape en inglés) (Feeney y Rainey, 2010).

Por último, las empresas sociales tienen una doble misión: económica y social. Solucionan un problema social mediante una actividad económica, a pesar de que, a veces, puede haber problemas asociados de maximización de ambos objetivos (Doherty et al., 2014). Estas empresas se caracterizan por tener unos valores sociales, democráticos, de interés por la comunidad y solidarios. La financiación de estas empresas procede tanto de la venta de bienes y servicios como de ayudas y financiación alternativa por parte de socios, terceras partes o de la administración pública. Aunque la administración pública muchas veces no proporcione ayudas económicas directas, si lo hace indirectamente, como con un menor gravamen fiscal. En este tipo de organizaciones, el modelo de stakeholders de Freeman (Freeman, 1984) adquiere todo su sentido, puesto que las empresas sociales son mucho más inclusivas que otro tipo de organizaciones a la hora de diseñar prácticas y políticas. Los trabajadores en este tipo de organizaciones como las cooperativas o empresas autogestionadas tienen más poder de decisión que en otras empresas. Esto puede hacer que los trabajadores de las empresas sociales presentan una mayor implicación moral a partir del trabajo que hacen (Kalleberg et al., 2006), y por tanto se podría relacionar con una mayor orientación eudaimónica en su bienestar. De hecho, investigaciones anteriores muestran que la participación en este tipo de empresa incrementa el bienestar eudaimónico, puesto que aumenta la confianza, las relaciones interpersonales, o el aprecio social (Tedmanson y Guerin, 2011).

Del mismo modo, los trabajadores de las empresas sociales sienten más continuidad puesto que estas organizaciones ofrecen trabajos estables y los trabajadores se pueden sentir más realizados (Pérotin, 2013). Además, una revisión reciente (Roy, Donaldson, Baker, y Kerr, 2014) apunta que las empresas sociales contribuyen al bienestar de una manera más global tanto para los individuos como para la sociedad. En concreto los trabajadores y usuarios incrementaban la salud física, la salud mental y la salud comunitaria, mediante el aumento de la dignidad, la creación de oportunidades o la interacción con la comunidad, proporcionando reconocimiento social y un ambiente de apoyo social.

Las empresas sociales están destinadas a satisfacer las necesidades de los miembros con una estructura democrática, de forma que los empleados puedan sentirse más vinculados con los propósitos de la empresa. Tal y como ponen de manifiesto las aportaciones de la democracia industrial (Heller, Pusic, Strauss, y Wilpert, 1998), la participación de trabajadores en la gestión y propiedad de las organizaciones puede hacer incrementar tanto la productividad como 
el bienestar de los trabajadores satisfaciendo tres necesidades básicas: incrementando la autonomía, la atención llena (o mindfulness) y reduciendo el aislamiento. De hecho, investigaciones previas muestran que los trabajadores de las empresas sociales son más felices y están más motivados por el significado de su trabajo y por el proyecto personal que desarrollan incluso cuando la recompensa económica es menor (Light, 2002). La autonomía también juega un papel muy importante. El hecho de ser propietario de la organización, como por ejemplo en cooperativas, da cierto poder de decisión y autonomía que podría estar ligado, según la teoría de la autodeterminación, a un bienestar más eudaimónico, una mayor satisfacción con la vida, y a una motivación más intrínseca (Ryan y Deci, 2001).

Por lo tanto, aunque no obtengan recompensas como los trabajadores de las empresas con ánimo de lucro (puesto que la distribución de beneficios es limitada), la satisfacción laboral y las recompensas intrínsecas son algunas de las variables que se han encontrado más elevadas en este tipo de organizaciones (Doherty et al., 2014).

En definitiva, yendo más allá del foco que existe actualmente en las empresas con ánimo de lucro, hemos visto como diferentes tipos de forma jurídica se asocian más estrechamente a diferentes tipos de cultura, valores o capacidades de gestión de los recursos humanos, y todo esto nos hace pensar que también habrá diferencias en el bienestar de sus trabajadores y en sus orientaciones hacia el bienestar. De esta manera, se contribuye a la literatura sobre la importancia del contexto en la gestión de los recursos humanos y en su bienestar. Teniendo en cuenta las diferencias analizadas entre los tres tipos de organización analizados, el siguiente objetivo de este trabajo es analizar si la forma jurídica de las organizaciones juega un papel relevante en el bienestar de los trabajadores (medido con diferentes indicadores).

\section{Metodología}

\section{Procedimiento y diseño}

El equipo de investigación contactó con varias organizaciones a través de sus gerentes o del gerente de RRHH, a los que se les pidió su colaboración para poder pasar cuestionarios a sus empleados. Con este objetivo, se les proporcionó información sobre los objetivos del proyecto, el procedimiento, el tiempo necesario de dedicación, etc. Finalmente se pidió la colaboración a cada uno de los empleados para que respondieran al cuestionario. La elección de la muestra fue por conveniencia, aunque se buscó que ésta fuera heterogénea con distintos sectores y ciudades españolas. Los empleados contestaron a los cuestionarios en horario laboral y mediante tres vías alternativas: en papel, en Tablet o de manera on-line con links personalizados. Todos los datos fueron agregados en una misma base de datos en SPSS para su posterior explotación. Para contrarrestar la posible varianza del método común, se procedió también a preguntar a los supervisores de los empleados que contestaron al cuestionario, los que respondieron un cuestionario en el que evaluaban el grado de satisfacción con el trabajo de cada uno de sus subordinados. Se utilizaron códigos internos para casar los trabajadores con sus respectivos supervisores (solo uno por trabajador). La participación fue voluntaria y confidencial. Este trabajo, por lo tanto, responde a un estudio descriptivo de poblaciones mediante encuestas con muestras probabilísticas de diseño transversal.

\section{Participantes}

Un total de 1568 trabajadores de 32 empresas de España participaron. Los trabajadores de las entidades públicas fueran los más numerosos (656 trabajadores), seguidos por los de las empresas con ánimo de lucro (641) y por último por los trabajadores de las empresas sociales (271). La mayor parte se dedican al sector 
terciario $(81,3 \%)$, y el resto al sector secundario (18,7\%). Algo más de la mitad de la muestra son mujeres $(54,6 \%)$. La mayoría de los participantes tienen edades comprendidas entre los 35 y los 50 años $(56,8 \%)$, seguidos por los menores de 35 años $(27 \%) \mathrm{y}$, finalmente, por los mayores de 50 años $(16,2 \%)$. Por último, la gran mayoría de los trabajadores tenían un trabajo a tiempo completo $(86 \%)$ y el resto $(14 \%)$ a tiempo parcial. Más información sobre la muestra según el tipo de forma jurídica se puede encontrar en la siguiente tabla 1.

Tabla 1. Características de la muestra por tipo de forma jurídica

\begin{tabular}{lccc}
\hline & $\begin{array}{c}\text { Ánimo de } \\
\text { lucro }\end{array}$ & Pública & Social \\
\hline Edad & & & \\
$<35$ & $36.2 \%$ & $11.7 \%$ & $41.6 \%$ \\
$35-50$ & $51.9 \%$ & $64.4 \%$ & $51.9 \%$ \\
$>50$ & $11.9 \%$ & $23.9 \%$ & $6.5 \%$ \\
\hline Género & & & \\
Femenino & $42.1 \%$ & $60.7 \%$ & $68 \%$ \\
Masculino & $57.9 \%$ & $39.3 \%$ & $32 \%$ \\
\hline Nivel Académico & & & \\
Universidad & $45.7 \%$ & $42.2 \%$ & $72.5 \%$ \\
Instituto & $14.4 \%$ & $33.7 \%$ & $10.2 \%$ \\
FP & $21 \%$ & $13.4 \%$ & $10.8 \%$ \\
Estudios básicos & $17.5 \%$ & $9.9 \%$ & $5.7 \%$ \\
Sin estudios & $1.4 \%$ & $.8 \%$ & $.8 \%$ \\
\hline
\end{tabular}

\section{IntstFlahoreciótdsopia.}

Satisfacción laboral. La satisfacción laboral se midió con la escala de Warr, Cook y Wall (IJSS: 1979) que incluye ítems sobre satisfacción laboral intrínseca y extrínseca. Un ejemplo de ítem es: "Indique en qué medida se siente satisfecho con la libertad de elegir su propia metodología de trabajo". La escala fue de tipo Likert de 7 puntos $(1=$ bastante insatisfecho y 7 = bastante satisfecho). El alfa de Cronbach es $\alpha=.85$.

Satisfacción con la vida. Para medir la satisfacción con la vida, utilizamos la escala de Diener, Emmons, Larsen y Griffin (1985). Un ejemplo de ítem es: "Las condiciones de mi vida son excelentes". La escala de respuesta fue de tipo Likert de 7 puntos $(1=$ completamente en desacuerdo y $7=$ completamente de acuerdo). El alfa de Cronbach es $\alpha=.82$.

Bienestar eudaimónico. Para medir el bienestar eudaimónico (denominado también bienestar psicológico) se utilizaron dos dimensiones de la escala de Ryff (1989), propósito en la vida y crecimiento personal. Un ejemplo de ítem es: "Tengo la sensación de que, con el tiempo, me he desarrollado mucho como persona". La escala de respuesta fue de tipo Likert con 7 puntos $(1=$ totalmente en desacuerdo y $7=$ totalmente de acuerdo). El alfa de Cronbach es $\alpha=.72$.

Orientación al bienestar. Se utilizaron dos dimensiones de la escala de Peterson, Park y Seligman (2005) para medir las orientaciones tanto hedónica como eudaimónica. Un ejemplo de ítem hedónico es: "la vida es demasiado corta para posponer los placeres que pueda proporcionar". Un ejemplo de ítem eudaimónico es: "Me siento responsable de hacer del mundo, un lugar mejor". La escala de respuesta fue de tipo Likert de 5 puntos $(1=$ nada y $5=$ mucho $)$. El alfa de Cronbach para la orientación eudaimónica es $\alpha=.72$ y para la hedónica $\alpha=.76$.

Satisfacción evaluada por el supervisor. La satisfacción evaluada por el supervisor se midió mediante un cuestionario ad-hoc con tres preguntas que los supervisores contestaban según la satisfacción que creían que tenían sus subordinados. Un ejemplo de ítem es: “¿Cree que el trabajador está satisfecho con el salario y las 
compensaciones que recibe por su trabajo?" La escala de respuesta fue de tipo Likert de 7 puntos ( 1 = muy insatisfecho y $7=$ muy satisfecho). El alfa de Cronbach es $\alpha=.69$.

Formajurídica. Para codificar esta variable utilizamos la forma jurídica de cada una de las empresas participantes y las agruparemos en 3 categorías: Entidades públicas (gobiernos locales y provinciales y escuelas públicas), entidades con ánimo de lucro (sociedades anónimas y limitadas) y empresas sociales (cooperativas, empresas de inserción y asociaciones con actividad económica).

\section{Análisis}

Primeramente, se calcularon los descriptivos y las correlaciones entre las variables. Después, se analizó mediante ANOVAs si las diferencias en las medias de cada uno de los indicadores de bienestar y orientaciones a la felicidad para los tres tipos de organizaciones eran significativas. Finalmente, se calcularon los análisis post-hoc para la diferencia de medias. Esto nos permite ver entre qué tipos de organización había diferencias significativas para cada tipo de bienestar. Todos los análisis se realizaron con el programa SPSS v.24.

\section{Resultados}

Los resultados muestran que, en general, los niveles de bienestar para las tres formas jurídicas son bastante elevados. Las medias superan en todos los casos la puntuación media de la escala y las desviaciones típicas son bastante pequeñas, lo que muestra poca variabilidad dentro de cada tipo de organización. Aun así, se puede ver que las medias no son iguales para las tres formas jurídicas (información mostrada en la tabla 2).

Una vez calculadas las medias, se procedió a analizar si éstas eran estadísticamente diferentes entre los tres grupos. Para esto se realizaron una serie de análisis de varianza. Se encontraron diferencias significativas para la satisfacción laboral en general $(\mathrm{F}=4.03 ; p=.018)$ como con la satisfacción laboral intrínseca $(\mathrm{F}=7.40 ; p=.001)$ y extrínseca $(\mathrm{F}=7.23 ; p=.001)$ y también para la satisfacción con la vida $(\mathrm{F}=6.69 ; p=.001)$. Del mismo modo se encontraron diferencias con el bienestar eudaimónico $(\mathrm{F}=13.88 ; p=.001)$. En cuanto a las orientaciones, se han encontrado diferencias significativas para la orientación hedónica $(\mathrm{F}=12.25 ; p=.001)$ y para la orientación eudaimónica $(\mathrm{F}=29.38 ; p=.001)$. Finalmente, se encontraron diferencias significativas con la satisfacción laboral evaluada por el supervisor $(\mathrm{F}=10.95 ; p=.001)$. Es decir, en general se encontraron diferencias significativas para cada una de las diferentes variables de bienestar (antes de las comparaciones entre las tres formas jurídicas mediante análisis post-hoc).

Como se puede ver en la tabla 2, los trabajadores de las empresas sociales son aquellos que presentan medias más elevadas en la mayor parte de indicadores de bienestar, como por ejemplo en la satisfacción laboral general. Pero, si analizamos separadamente cada dimensión de la satisfacción laboral, comprobamos que los trabajadores de las empresas con ánimo de lucro son los que más puntúan en satisfacción laboral extrínseca. Los trabajadores de las empresas sociales también puntúan más en satisfacción con la vida, bienestar eudaimónico y satisfacción según los supervisores. Finalmente, en cuanto a las orientaciones, los trabajadores de las empresas con ánimo de lucro presentan una mayor orientación hedónica, seguidos de los trabajadores públicos. Al contrario pasa con la orientación eudaimónica, donde los trabajadores de las empresas sociales presentan valores mayores, seguidos de los trabajadores públicos y por último los trabajadores de las empresas con ánimo de lucro.

Tabla 2. Media y desviación típica de las variables 


\begin{tabular}{lcccccc}
\hline & \multicolumn{2}{c}{ Ánimo de lucro } & \multicolumn{2}{c}{ Pública } & \multicolumn{2}{c}{ Social } \\
\hline Satisfacción laboral & & DT & & DT & & DT \\
\multicolumn{1}{c}{ Sat. Laboral Intrínseca } & 5.17 & .95 & 5.07 & .91 & 5.24 & .89 \\
\multicolumn{1}{c}{ Sat. Laboral Extrínseca } & 5.16 & 1.08 & 5.14 & 1.10 & 5.42 & .98 \\
Satisfacción con la vida & 5.07 & 1.02 & 4.98 & .99 & 5.04 & .99 \\
Bienestar eudaimónico & 5.75 & .71 & 5.76 & .80 & 6.02 & .66 \\
Orientación hedónica & 3.56 & .84 & 3.39 & .77 & 3.31 & .79 \\
Orientación eudaimónica & 3.18 & .89 & 3.49 & .76 & 3.53 & .77 \\
Sat. Evaluado supervisor & 5.14 & .96 & 5.07 & .91 & 5.45 & .95 \\
\hline
\end{tabular}

Nota: Elaboración Propia.

Seguidamente, para comprobar entre qué tipos de organizaciones las diferencias eran significativas se llevaron a cabo análisis posthoc, usando el test de Tukey. Los resultados se encuentran en la tabla 3 , donde se comparan para cada variable de bienestar, las medias de cada pareja de tipo de organización, y si esa diferencia de medias es significativa o no.

Tabla 3. Análisis post-hoc de diferencia de medias

Tabla 3. Análisis post-hoc de diferencia de medias

\begin{tabular}{|c|c|c|c|c|c|}
\hline \multirow{5}{*}{\multicolumn{3}{|c|}{$\begin{array}{l}\text { Significativas se llevaron a cabo análisis post- } \\
\text { hoc, usando el test de Tukey. Los resultados se } \\
\text { encuentran en la tabla } 3 \text {, donde se comparan para } \\
\text { cada variable de bienestar, las medias de cada } \\
\text { pareja de tipo de organización, y si esa diferencia } \\
\text { de medias es significativa o no. }\end{array}$}} & \multirow{3}{*}{$\begin{array}{l}\text { Sat. Vida }{ }_{\text {pública }} v s \text { Sat. Vida } \\
\text { Eucial } \\
\text { Eudaimónico }_{\text {lucro }} v s \\
\text { Eudaimónico }_{\text {pública }}\end{array}$} & \multirow{2}{*}{$\begin{array}{l}5.18=5.34 \\
5.75=5.76\end{array}$} & \multirow{2}{*}{$\frac{.097}{.98}$} \\
\hline & & & & & \\
\hline & & & & & \\
\hline & & & Eudaimónico $_{\text {social }}$ & $0.15<0.02$ & .001 \\
\hline & & & $\begin{array}{l}\text { Eudaimónico }_{\text {pública }} v s \\
\text { Eudaimónico }_{\text {social }}\end{array}$ & $5.76<6.02$ & .001 \\
\hline \multicolumn{3}{|c|}{ Tabla 3. Análisis post-hoc de diferencia de medias } & $\begin{array}{l}\text { O.hedónica }_{\text {lucro }} v s \\
\text { H.hedónica }_{\text {pública }}\end{array}$ & $3.56>3.39$ & .001 \\
\hline Post-hoc análisis & Media & $p$ & $\begin{array}{l}\text { O.hedónica }_{\text {lucro }} v s \\
\text { H.hedónica }_{\text {social }}\end{array}$ & $3.56>3.31$ & .001 \\
\hline $\begin{array}{l}\text { Sat. Laboral } \\
\text { Laboral }_{\text {pública }} \text { vs Sat. }\end{array}$ & $5.17=5.07$ & .096 & $\begin{array}{l}\text { O.hedónica } \\
\text { H.hedíblica } \\
\text { H.hica }\end{array}$ & $3.39=3.31$ & .396 \\
\hline $\begin{array}{l}\text { Sat. Laboral } \\
\text { Laboral }_{\text {lucial }} \text { vs Sat. }\end{array}$ & $5.17=5.24$ & .629 & $\begin{array}{l}\text { O. eudaimónica }{ }_{\text {lucro }} v s \mathrm{H} . \\
\text { eudaimónica }\end{array}$ & $3.18<3.49$ & .001 \\
\hline $\begin{array}{l}\text { Sat. Laboral } \\
\text { Laborblica }_{\text {social }} \text { vs Sat. }\end{array}$ & $5.07<5.24$ & .025 & $\begin{array}{l}\text { O. eudaimónica }{ }_{\text {lucro }} v s \mathrm{H} \text {. } \\
\text { eudaimónica }_{\text {social }}\end{array}$ & $3.18<3.53$ & .001 \\
\hline Intrínseco ${ }_{\text {lucro }} v s$ Intrínseco ${ }_{\text {pública }}$ & $5.16=5.14$ & .955 & \multirow{2}{*}{$\begin{array}{l}\text { O. eudaimónica } \\
\text { eública } \\
\text { eudaimónica } \\
\text { social }\end{array}$} & \multirow{2}{*}{$3.49=3.53$} & \multirow{2}{*}{.837} \\
\hline Intrínseco $_{\text {lucro }} v S$ Intrínseco social $_{\text {. }}$ & $5.16<5.42$ & .002 & & & \\
\hline Intrínseco $_{\text {pública }} v S$ Intrínseco ${ }_{\text {social }}$ & $5.14<5.42$ & .001 & \multirow{3}{*}{$\begin{array}{l}\text { Sat. supervisor } \\
\text { supervisoro }_{\text {pública }} \text { vs Sat. } \\
\text { Sat. supervisor }_{\text {lucro }} \text { vs Sat. } \\
\text { supervisor }_{\text {social }}\end{array}$} & \multirow{2}{*}{$5.14=5.07$} & .684 \\
\hline Extrínseco $_{\text {lucro }} v s$ Extrínseco ${ }_{\text {pública }}$ & $5.19>4.98$ & .001 & & & \multirow{2}{*}{.001} \\
\hline Extrínseco $_{\text {lucro }} v s$ Extrínseco $_{\text {social }}$ & $5.19=5.04$ & .105 & & $5.14<5.45$ & \\
\hline $\begin{array}{l}\text { Extrínseco }_{\text {pública }} v s \\
\text { Extrínseco }_{\text {social }}\end{array}$ & $4.98=5.04$ & 673 & $\begin{array}{l}\text { Sat. supervisor } \\
\text { pública } \\
\text { supervisor }_{\text {social }} \text { Sat. }\end{array}$ & $5.07<5.45$ & .001 \\
\hline Sat. Vida lucro $_{\text {vora }}$ Sat. Vida pública & $5.07=5.18$ & .114 & \multicolumn{3}{|c|}{ Estos resultados muestran que, respecto a la } \\
\hline Sat. Vida ${ }_{\text {lucro }} v S$ Sat. Vida $a_{\text {social }}$ & $5.07<5.34$ & .001 & Nota: Elaboración Propia. & & \\
\hline
\end{tabular}


satisfacción laboral general, se han encontrado diferencias significativas entre las organizaciones públicas y las sociales, siendo éstas últimas las que reflejaban mayor nivel de bienestar. Si analizamos los resultados por tipos de satisfacción laboral, vemos como los trabajadores de las empresas sociales reportan niveles más elevados de satisfacción intrínseca comparado con los trabajadores de las entidades públicas y de las de ánimo de lucro. En cambio, para la satisfacción extrínseca, aquellos que reportan mayores niveles son, precisamente, los trabajadores de las empresas con ánimo de lucro comparados con los trabajadores de las organizaciones públicas. La única comparación significativa con la satisfacción con la vida ha sido entre empresas sociales (con resultados más elevados) y empresas con ánimo de lucro. En cuanto al bienestar eudaimónico, se ha podido comprobar que, los trabajadores de las empresas sociales han reportado mayores niveles de bienestar comparando con los trabajadores de las empresas con ánimo de lucro y las públicas. Finalmente, cuando la satisfacción laboral es evaluada por el supervisor también se encuentran diferencias significativas entre las empresas sociales (con mayor puntuación) comparado con los otros dos grupos.

En cuanto a las orientaciones hacia la felicidad, los trabajadores de las empresas con ánimo de lucro son aquellos que tienen una orientación más pronunciada hacia el hedonismo, comparado con los otros dos grupos; mientras que son estos trabajadores también los que reportan menos orientación eudaimónica cuando los comparamos con los trabajadores de las empresas sociales y las públicas. A continuación, se discuten estos resultados con la teoría previamente analizada.

\section{Discusión}

El objetivo de este trabajo era analizar si la forma jurídica de las organizaciones, teniendo en cuenta las características, valores, expectativas que producen en sus empleados, etc., juega un papel importante en el bienestar de los trabajadores, operacionalizado según distintos indicadores. En una muestra de trabajadores de empresas con ánimo de lucro, públicas y sociales, se han encontrado diferencias en varios indicadores de bienestar y orientaciones a la felicidad. Dichos resultados muestran apoyo a la teoría institucional y la consideración que las distintas demandas internas y externas de cada tipo de organización facilitan el desarrollo de distintos tipos de valores (orientaciones a la felicidad) y resultados laborales (DiMaggio y Powell, 1983).

Los análisis de varianza muestran diferencias significativas en todos los indicadores estudiados: bienestar hedónico (satisfacción laboral intrínseca y extrínseca y satisfacción con la vida), bienestar eudaimónico, orientaciones a la felicidad y satisfacción evaluada por el supervisor. Mediante varios análisis post-hoc, hemos podido comprobar que estas diferencias se daban sobre todo entre los trabajadores de las empresas sociales y los de las empresas con ánimo de lucro. También hemos encontrado algunas diferencias entre los trabajadores de empresas públicas y sociales, y ya en menor medida entre públicas y con ánimo de lucro. Estas diferencias encontradas empíricamente responden a aquellas discrepancias que hemos analizado según la financiación, la misión, y gestión de recursos humanos entre los tres tipos de organización (Doherty et al., 2014).

Uno de los resultados más interesantes hace referencia a las diferencias respecto a las orientaciones a la felicidad. Los trabajadores de las empresas con ánimo de lucro tienen una mayor orientación hacia el bienestar hedónico, hacia el placer y las recompensas. Este resultado indica información relevante para entender los valores de los trabajadores de las diferentes organizaciones y coincide con uno de los grandes pilares de las empresas con ánimo de lucro que es la búsqueda del beneficio económico. Si estas empresas, tienen a bien la repartición de beneficios económicos y de recompensar económicamente a los trabajadores, 
es lógico que los trabajadores presenten una orientación más hedónica y una satisfacción extrínseca mayor (Gkorezis y Petridou, 2012).

Por el contrario, los trabajadores de organizaciones sociales y públicas presentan mayor orientación hacia el bienestar eudaimónico que los trabajadores de las empresas con ánimo de lucro. Cómo hemos comentado anteriormente, las organizaciones públicas y sociales se centran en unos valores más sociales ayudando a incrementar el bienestar de sus usuarios y por tanto los trabajadores pueden sentir que su trabajo tiene un propósito que va más allá de su rutina diaria, coincidiendo con anteriores investigaciones (Light, 2002).

Estos resultados también estarían en línea con el modelo de ajuste cultural o el modelo de atracción, selección y atrición, al mostrar un alineamiento entre los trabajadores y las organizaciones en relación a los valores organizacionales (Schneider, et al., 2001). Es decir, los trabajadores de las empresas con ánimo de lucro, al presentar una orientación hedónica mayor, se pueden sentir más atraídos para trabajar en una organización con ánimo de lucro donde podrán satisfacer en mayor medida esta necesidad. De igual manera, los trabajadores de las empresas sociales, al presentar niveles más altos de orientación eudaimónica, se sentirían más atraídos por empresas con valores más sociales que les permitirían una mayor congruencia con sus propios valores.

Los resultados que obtenemos en relación con los diferentes indicadores de bienestar también parecen alineados con estas características y valores organizativos. De este modo, los trabajadores de empresas con ánimo de lucro presentan, no solo una mayor orientación hedónica, sino también una mayor satisfacción extrínseca. Por el contrario, los trabajadores de las empresas sociales, tienen una mayor orientación eudaimónica que los de las empresas con ánimo de lucro, y también perciben mayor satisfacción intrínseca. Las orientaciones al bienestar guían los comportamientos de los trabajadores. Cuando estos actúan de acuerdo con sus orientaciones tienen más probabilidades de tener experiencias positivas (Huta, 2015). Aun así, hay que tener en cuenta que lo que se evalúa en la presente investigación son los valores expresados por los propios miembros de las organizaciones, que pueden responder en mayor medida a los valores de la cultura deseada (personal o corporativa) que a los propios valores o comportamientos personales (Roccas y Sagiv, 2010). Aun así, la literatura ha mostrado en repetidas ocasiones relaciones positivas entre los valores expresados y la conducta personal (de Groot y Steg, 2008; Feather, 1995). Asimismo, estos resultados pueden estar influenciados por otras variables. En el caso de las personas con mayor nivel académico, se ha mostrado que estos trabajadores perciben mayores niveles de bienestar (Keyes, Shmotkin, y Ryff, 2002). Recientes investigaciones han encontrado resultados parecidos donde las mujeres reportaban niveles más elevados de satisfacción en la vida $\mathrm{y}$ en el trabajo que los hombres (Al-Attiyah y Nasser, 2016). Como se ha visto en los datos demográficos, los empleados de las empresas sociales presentan un nivel más alto de formación y una mayor proporción de mujeres, lo que podría estar alterando estos resultados.

Incluir distintos indicadores de bienestar que consideran otros aspectos más allá de la satisfacción laboral (Guest, 2002; Warr y Nielsen, 2018) nos da una perspectiva más cuidadosa de la relación entre el tipo de organización y el bienestar. Así, se pone de manifiesto la importancia de incluir variables como la satisfacción con la vida (Della Torre, 2012), el bienestar eudaimónico (Ryan y Deci, 2001) o las orientaciones hacia el bienestar (Huta y Waterman, 2014). Por lo tanto, el bienestar de los trabajadores es una variable mucho más compleja que alcanza indicadores más allá de la satisfacción laboral.

También ha quedado patente, como el contexto, en este caso la forma jurídica, es una 
variable a tener en cuenta cuando se analiza la gestión de los recursos humanos (Paauwe, 2004), puesto que tiene un efecto diferenciador en los tipos de bienestar de los trabajadores. Las diferencias existentes en los niveles de bienestar entre las distintas formas jurídicas analizadas ponen de manifiesto la necesidad de ampliar los estudios en el ámbito del management y la psicología organizativa a otras poblaciones, más allá de las empresas con ánimo de lucro (Feeney y Rainey, 2010). La teoría institucional, ofrece un marco de referencia adecuado para entender cómo las diferentes formas jurídicas representan distintos valores culturales (Cameron y Quinn, 2011) y formas de gestionar las organizaciones (Day et al., 2014), que afectan de forma diferencial a distintos indicadores de bienestar de sus trabajadores (Medina, 2012).

\section{Limitaciones y futuras investigaciones}

Esta investigación no está carente de limitaciones. La varianza del método común puede ser un problema en la investigación conductual (Podsakoff, MacKenzie, Lee, y Podsakoff, 2003); sin embargo, las medidas de autoinforme son necesarias cuando se considera la información sobre los estados internos, como el bienestar. Además, hemos añadido medidas del supervisor para contrarrestar esta problemática. En segundo lugar, el presente trabajo tiene una muestra desigual para las tres formas jurídicas, ya que la conforman 656 trabajadores de entidades públicas, 641 de empresas con ánimo de lucro y 271 de empresas sociales. Futuras investigaciones deberían de ampliar la muestra de trabajadores de empresas sociales para poder comparar en la mejor de las condiciones los tres tipos de empresas. Además, como se ha visto, el 81,3\% de las empresas se dedicaban al sector servicios o terciario, por lo que también sería interesante ampliar la muestra a organizaciones del sector secundario, ya que estas diferencias también podrían influir en el bienestar de los trabajadores.
Otra limitación de este estudio es la marcada influencia legislativa española. Esto es, las formas jurídicas se han establecido a partir de la tipología según la jurisprudencia española. En otros países pueden existir formas jurídicas adicionales que no se han tenido en cuenta. Además, tampoco hemos considerado a las ONGs por ser un tipo de organización muy particular y sin actividad económica. No obstante, creemos que la diferenciación realizada abarca a la mayoría de organizaciones, y contribuye a la literatura en cuanto a añadir a las empresas sociales a la dicotomía público-privado.

Con el compromiso de continuar estudiando la variable de la forma jurídica, futuras investigaciones deberían estudiar su influencia sobre otros resultados organizativos como por ejemplo el grado de compromiso con la organización o el rendimiento, o su efecto modulador sobre las relaciones entre determinadas prácticas organizativas y los resultados laborales. Sería también interesante estudiar la relación entre el ajuste organización-persona (en términos de valores u orientaciones a la felicidad) y su relación con el bienestar. De hecho, la hipótesis del ajuste ambiente-valores mantiene que, en la medida que los valores de un trabajador son congruentes con los de la organización, mayor será el grado de satisfacción y compromiso de éste con su trabajo.

\section{Conclusiones}

El presente estudio pone de relieve la importancia de la forma jurídica como variable del contexto en el estudio del bienestar y para la gestión de las personas y las organizaciones. Las diferencias en los objetivos que persiguen, su cultura y valores y las propias expectativas que generan llevan a resultados distintos en su gestión y los resultados organizacionales.

La literatura sobre el bienestar se ha 
focalizado tradicionalmente en empresas con ánimo de lucro. No obstante, la importancia de comparar estos resultados con otro tipo de organizaciones nos ha llevado a utilizar también empresas sociales y entidades públicas, dando resultados muy importantes. Trabajar en una empresa con ánimo de lucro, pública o social tiene consecuencias sobre los niveles de bienestar que reportan los trabajadores. Además, se ha puesto de manifiesto la importancia de atender distintos aspectos o dimensiones del bienestar laboral, donde hemos podido combinar dimensiones de bienestar hedónico con dimensiones eudaimónicas y diferentes orientaciones a la felicidad. Todo esto nos permite corroborar como, en diferentes tipos de empresa (empresas con ánimo de lucro, entidades públicas y empresas sociales), las orientaciones a la felicidad y el tipo de bienestar son significativamente diferentes.

\section{Referencias}

Al-Attiyah, A., y Nasser, R. (2016). Gender and age differences in life satisfaction within a sexsegregated society: Sampling youth in Qatar. International Journal of Adolescence and Youth, 21(1), 84-95. https://doi.org/10.1080/02673843.2013.8 08158

Aycan, Z., Kanungo, R. N., y Sinha, J. B. P. (1999). Organizational culture and human resource management practices. Journal of Cross-Cultural Psychology, 30(4), 501-526. https://doi. org/10.1177/0022022199030004006

Aycan, Z., Kanungo, R., Mendonca, M., Yu, K., Deller, J., Stahl, G., y Kurshid, A. (2000). Impact of culture on human resource management practices: A 10-country comparison. Applied Psychology, 49(1), 192-221. doi:10.1111/1464-0597.00010

Boon, C., den Hartog, D. N., Boselie, P., y
Paauwe, J. (2011). The relationship between perceptions of HR practices and employee outcomes: Examining the role of person-organisation and personjob fit. International Journal of Human Resource Management, 22(1), 138-162. doi: 10.1080/09585192.2011.538978

Cameron, K., y Quinn, R. (2011). Diagnosing and changing organizational culture: Based on the competing values framework (3a edición). San Francisco, CA: Jossey-Bass.

Cottini, E., y Lucifora, C. (2013). Mental health and working conditions in Europe. ILR Review, 66(4), 958-988. https://doi. org/10.1177/001979391306600409

Day, A., Kelloway, E. K., y Hurrell, J. J. (2014). Workplace well-being: How to build psychologically healthy workplaces. West Sussex, Reino Unido, Wiley-Blackwell.

de Groot, J., y Steg, L. (2008). Value Orientations to Explain Beliefs Related to Environmental Significant Behavior. Environment and Behavior, 40(3), 330354. doi: 10.1177/0013916506297831

Della Torre, E. (2012). High performance work systems and workers' well-being: A sceptical view. International Journal of Work Innovation, 1(1), 7-23. doi: 10.1504/ IJWI.2012.047974

Den Hartog, D. N., Boselie, P., y Paauwe, J. (2004). Performance management: A model and research agenda. Applied Psychology: An international review, 53(4), 556-569. http://dx.doi.org/10.1111/ j.1464-0597.2004.00188.x

Diener, E., Emmons, R. A., Larsen, R. J., y Griffin, S. (1985). The satisfaction with life scale. Journal of Personality Assessment, 49(1), 71-75. https://doi.org/10.1207/ s15327752jpa4901_13 
Diener, E., y Lucas, R. E. (1999). Personality and subjective well-being. En Kahneman, D., Diener, E., y Schwartz, N. (Eds), Well-being: The foundations of hedonic psychology (pp. 213-229). Nueva York: Russell Sage

DiMaggio, P. J., y Powell, W. W. (1983). The iron cage revisited: Institutional isomorphism and collective rationality in organizational fields. American Sociological Review, 48(2), 147-160. doi: 10.2307/2095101

Doherty, B., Haugh, H., y Lyon, F. (2014). Social enterprises as hybrid organizations: A review and research agenda. International Journal of Management Reviews, 16(4), 417-436. https://doi.org/10.1111/ ijmr. 12028

Doolen, T., Hacker, M., y Van Aken, E. (2003). The Impact of Organizational Context on Work Team Effectiveness: A Study of Production. IEEE Transactions on engineering management, 50(3), 285-296 doi: 10.1109/TEM.2003.817296

Feather, N. T. (1995). Values, valences, and choice: The influence of values on the perceived attractiveness and choice of alternatives. Journal of Personality and Social Psychology, 68, 1135-1151. http:// dx.doi.org/10.1037/0022-3514.68.6.1135

Feeney, M. K., y Rainey, H. G. (2010). Personnel flexibility and red tape in public and nonprofit organizations: Distinctions due to institutional and political accountability. Journal of Public Administration Research and Theory, 20(4), 801-826. https://doi. org/10.1093/jopart/mup027

Fletcher, T. D., Major, D. A., y Davis, D. D. (2008). The interactive relationship of competitive climate and trait competitiveness with workplace attitudes, stress, and performance. Journal of Organizational Behavior, 29(7), 899-922. http://dx.doi. org/10.1002/job.503

Freeman, R. (1984). Strategic management. A stakeholder approach. Londres: Pitman.

Gkorezis, P., y Petridou, E. (2012). The effect of extrinsic rewards on public and private sector employees' psychological empowerment: A comparative approach. The International Journal of Human Resource Management, 23(17), 35963612. https://doi.org/10.1080/09585192.2 011.639025

Guest, D. (2002). Human resource management, corporate performance and employee wellbeing: Building the worker into HRM. Journal of Industrial Relations, 44(3), 335-358. https://doi.org/10.1111/1472$\underline{9296.00053}$

Guest, D. (2017). Human resource management and employee well-being: towards a new analytic framework. Human Resource Management Journal, 27, 22-38. doi: 10.1111/1748-8583.12139.

Heller, F., Pusic, E., Strauss, G., y Wilpert, B. (1998). Organisational Participation: Myth and Reality. Oxford: Oxford University Press.

Henderson, L. W., Knight, T., y Richardson, B. (2014). The Hedonic and Eudaimonic Validity of the Orientations to Happiness Scale. Social Indicators Research, 115(3), 1087-1099. doi: 10.1007/s11205-0130264-4

Huta, V. (2015). An overview of hedonic and eudaimonic well-being concepts. En L. Reinecke y M. B. Oliver (Eds.), The Routledge handbook of media use and well-being: International perspectives 
on theory and research on positive media effects. (pp. 14-33). Nueva York: Routledge/Taylor \& Francis Group

Huta, V., y Waterman,A.S. (2014). Eudaimonia and its distinction from hedonia: Developing a classification and terminology for understanding conceptual and operational definitions. Journal of Happiness Studies, 15(6), 1425-1456. doi: 10.1007/s10902013-9485-0

Irvine, D. M., y Evans, M. G. (1995). Job satisfaction and turnover among nurses: Integrating research findings across studies. Nursing Research, 44(4), 246253. http://dx.doi.org/10.1097/00006199199507000-00010

Judge, T. A., Thoresen, C. J., Bono, J. E., y Patton, G. K. (2001). The job satisfaction-job performance relationship: A qualitative and quantitative review. Psychological Bulletin, 127(3), 376-407. Doi: I0.1037//0033-2909.I27.3.376

Kalleberg, A. L., Marsden, P. V., Reynolds, J., y Knoke, D. (2006). Beyond profit? Sectoral differences in highperformance work practices. Work and Occupations, 33(3), 271-302. https://doi. org/10.1177/0730888406290049

Kanungo, R. N., y Aycan, Z. (1998). Issue of culture fit in human resource management practices. Vision, 2(1), 5-11. https://doi.or $\mathrm{g} / 10.1177 / 09722629 X 98002001002$

Keyes, C. L. M., Shmotkin, D., y Ryff, C. D. (2002). Optimizing well-being: The empirical encounter of two traditions. Journal of Personality and Social Psychology, 82(6), 1007-1022. Doi: 10.1037//00223514.82.6.1007

Light, P. (2002). The content of their character: The state of the nonprofit workforce.
Nonprofit Quarterly, 9(3), 6. Disponible en. https://nonprofitquarterly.org/thecontent-of-their-character-the-state-ofthe-nonprofit-workforce/

Macklin,D.S., Smith, L.A., y Dollard, M.F.(2006). Public and private sector work stress: Workers compensation, levels of distress and job satisfaction, and the demandcontrol-support model. Australian Journal of Psychology, 58(3), 130-143. https://doi. org/10.1080/00049530600940190

Medina, E. (2012). Job satisfaction and employee turnover intention: What does organizational culture have to do with it? Tesis de master. Columbia University.

Ostroff, C., Kinicki, A. J., y Tamkins, M. M. (2003). Organizational culture and climate. Handbook of psychology, Nueva Jersey, John Wiley and Sons, Inc.

Paauwe, J. (2004). HRM and performance: Unique approaches for achieving long term viability. Oxford: Oxford University Press.

Parent-Thirion, A., Fernández, E., Hurley, J., y Vermeylen, G. (2007). Fourth european working conditions survey. ( $\mathrm{N}^{\mathrm{o}} \mathrm{EF} / 06 / 98 /$ EN). Luxemburg: Office for Official Publications of the European Communities. Disponible en: publications.europa.eu

Peiró, J.M., Ayala, Y., Tordera, N., Lorente, L., y Rodríguez, I. (2014). Bienestar sostenible en el trabajo: Revisión y reformulación. Papeles del Psicólogo, 35, 7-16. Disponible en: http://www.papelesdelpsicologo.es/ pdf/2316.pdf

Pérotin, V. (2013). Worker cooperatives: Good, sustainable jobs in the community. Journal of Entrepreneurial and Organizational Diversity, 2(2), 34-47. Disponible en: 
https://ssrn.com/abstract $=2437929$

Peterson, C., Park, N., y Seligman, M. E. P. (2005). Orientations to happiness and life satisfaction: The full life versus the empty life. Journal of Happiness Studies, 6(1), 25-41. doi 10.1007/s10902-004-1278-z

Petrescu, A., y Simmons, R. (2008). Human resource management practices and workers' job satisfaction. International Journal of Manpower, 29(7), 651-667. Doi 10.1108/01437720810908947

Podsakoff, P. M., MacKenzie, S. B., Lee, J. Y., y Podsakoff, N. P. (2003). Common method biases in behavioral research: A critical review of the literature and recommended remedies. Journal of Applied Psychology, 88(5), 879-903. $\quad$ http://dx.doi. org/10.1037/0021-9010.88.5.879

Roccas, S. y Sagiv, L. (2010). Personal Values and Behavior: Taking the Cultural Context into Account. Social and Personality Psychology Compass, 4, 30-41. https://doi. org/10.1111/j.1751-9004.2009.00234.x

Roy, M. J., Donaldson, C., Baker, R., y Kerr, S. (2014). The potential of social enterprise to enhance health and well-being: A model and systematic review. Social Science \& Medicine, 123, 182-193, doi: 10.1016/j. socscimed.2014.07.031.

Ryan, R., y Deci, E. (2001). On happiness and human potentials: A review of research on hedonic and eudaimonic well-being. Annual Review of Psychology, 52, 141166. https://doi.org/10.1146/annurev. psych.52.1.141

Ryff, CD. (1989). Happiness is everything, or is it? Explorations on the meaning of psychological well-being. Journal of Personality and Social Psychology. 57:1069-81. http://dx.doi. org/10.1037/0022-3514.57.6.1069

Ryff, C., y Singer, B. (2008). Know thyself and become what you are: A eudaimonic approach to psychological well-being. Journal of Happiness Studies, 9(1), 13-39. doi 10.1007/s10902-006-9019-0

Schneider, B., Smith, D. B., y Paul, M. C. (2001). P-E fit and the attractionselection-attrition model of organizational functioning: Introduction and overview. En M. Erez, U. Kleinbeck, y H. Thierry (Eds.), Work motivation in the context of a globalizing economy (pp. 231-246). Mahwah, Nueva Jersey, Estados Unidos: Lawrence Erlbaum Associates Publishers.

Songfa, Z., Idan, S., David, K., Ebstein, R. P., y Chew, S. H. (2018). Competitiveness and stress. International Economic Review, 59, 1263-1281 doi:10.1111/iere.12303

Tarcan, G. Y., Tarcan, M., y Top, M. (2017). An analysis of relationship between burnout and job satisfaction among emergency health professionals. Total Quality Management and Business Excellence, 28(11-12), 1339-1356. https://doi.org/10. 1080/14783363.2016.1141659

Tedmanson, D., y Guerin, P. (2011). Enterprising social wellbeing: social entrepreneurial and strengths based approaches to mental health and wellbeing in "remote" Indigenous community contexts. Australas Psychiatry. 19(1), 30-33 doi: 10.3109/10398562.2011.583078.

Van de Voorde, K., Paauwe, J., y Van Veldhoven, M. (2012). Employee Well-Being and the HRM - organizational performance relationship: A review of quantitative studies. International Journal of Management Reviews, 14(4), 391-407. 
doi: $10.1111 / j .1468-2370.2011 .00322 . x$

Vandenabeele, W., Brewer, G. A., y Ritz, A. (2014). Past, present, and future of public service motivation research. Public Administration, 92(4), 779-789. doi: 10.1111/padm.12136

Warr, P., y Nielsen, K. (2018). Wellbeing and work performance. En E. Diener, S. Oishi y L. Tay (Eds.), Handbook of well-being (pp. 1-22). Salt Lake City: UT: DEF Publishers.

Warr, P. (2013). Fuentes de felicidad e infelicidad en el trabajo: Una perspectiva combinada.
Revista De Psicología Del Trabajo Y De Las Organizaciones, 29(3), 99-106. https://doi.org/10.5093/tr2013a15

Warr, P., Cook, J., y Wall, T. (1979). Scales for the measurement of some work attitudes and aspects of psychological well-being. Journal of Occupational Psychology, 52(2), 129-148. https://doi. org/10.1111/j.2044-8325.1979.tb00448.x

Wright, B. E., y Pandey, S. K. (2011). Public organizations and mission valence: When does mission matter? Administration and Society, 43(1), 22-44. doi: $10.1177 / 0095399710386303$ 